International Mathematical Forum, 2, 2007, no. 19, 919 - 928

\title{
On Schur complements of doubly diagonally dominant matrices and $H$-matrices ${ }^{1}$
}

\author{
Ting-Zhu Huang ${ }^{2}$, Gui-Xian Tian \\ School of Applied Mathematics \\ University of Electronic Science and Technology of China \\ Chengdu, Sichuan, 610054, P. R. China \\ Shu-Yu Cui \\ Department of Mathematics, Heze University \\ Heze, Shandong, 274015, P. R. China
}

\begin{abstract}
It is known that the Schur complements of $H$-matrices and doubly diagonally dominant matrices are closure. In this paper, we obtain that the same are true of their diagonal-Schur complements. Further, we study their distribution of eigenvalues.
\end{abstract}

Keywords: Diagonally dominant matrix; Doubly diagonally dominant matrix; $H$-matrix; Schur complement; Diagonal-Schur complement; Eigenvalue

Mathematics Subject Classification: 15A45; 15A48

\section{Introduction}

Given a matrix family, it is interesting to know whether some important properties or structures of the class of matrices are inherited by their submatrices or by the matrices associated with the original matrices. It is known that the principal submatrices and the Schur complements of positive semidefinite matrices are positive semidefinite matrices; the same is true of $M$-matrices, $H$-matrices, diagonally dominant matrices, doubly diagonally dominant matrices, and of inverse $M$-matrices (see [1-6]). J. Liu, Y. Huang [5] consider the

\footnotetext{
${ }^{1}$ Supported by NCET of China (NCET-04-0893), applied basic research foundation of Sichuan province (05JY029-068-2).

${ }^{2}$ Corresponding author. E-mail: tzhuang@uestc.edu.cn (T. Z. Huang)
} 
diagonal-Schur complements of strictly diagonally dominant matrices $\left(S D_{n}\right)$, strictly $\gamma$-diagonally dominant matrices $\left(S D_{n}^{\gamma}\right)$, strictly product $\gamma$-diagonally dominant matrices $\left(S P D_{n}^{\gamma}\right)$. They showed that the diagonal-Schur complements of $S D_{n}$ (or $S D_{n}^{\gamma}, S P D_{n}^{\gamma}$ ) inherit the structure property of the original matrices, and gave their distribution of eigenvalues. In this paper, we shall consider the diagonal-Schur complements of $H$-matrices and doubly diagonally dominant matrices.

Let $C^{m \times n}\left(R^{m \times n}\right)$ denote the set of $m \times n$ complex (real) matrices. For $A=\left(a_{i j}\right)$ and $B=\left(b_{i j}\right) \in R^{m \times n}$, we say $A \geq B$ if $a_{i j} \geq b_{i j}$ for all $i, j$. A real $n \times n$ matrix $A$ is called an $M$-matrix if $A=s I-B$, where $B \geq 0$ and $s>\rho(B)$ the spectral radius of $B$. Let $M_{n}$ denote the set of $n \times n M$ matrices, and $Z_{n}$ denote the set of $n \times n$ non-positive off-diagonal elements matrices. The comparison matrix of $A$, denoted by $\mu(A)=\left(\alpha_{i j}\right) \in C^{n \times n}$, is defined by $\alpha_{i j}=\left\{\begin{array}{ll}\left|a_{i i}\right| & i=j, \\ -\left|a_{i j}\right| & i \neq j .\end{array} \quad\right.$ A matrix $A \in C^{n \times n}$ is called an H-matrix if $\mu(A) \in M_{n}$. Let $H_{n}$ denote the set of $n \times n H$-matrix, the absolute matrix of $A$ is defined by $|A|=\left(\left|a_{i j}\right|\right)$. For $A=\left(a_{i j}\right)$ and $B=\left(b_{i j}\right) \in C^{m \times n}$, the Hadamard product of $A$ and $B$ is the matrix $\left(a_{i j} b_{i j}\right)$, denoted by $A \circ B$.

Let $N=\{1,2, \ldots, n\}$, if $\alpha \subseteq N,|\alpha|$ denote the cardinality of $\alpha$. For nonempty index sets $\alpha, \beta \subset N$, we denote by $A(\alpha, \beta)$ that submatrix of $A \in$ $C^{n \times n}$ lying in the rows indicated by $\alpha$ and the columns indicated by $\beta$. The submatrix $A(\alpha, \alpha)$ is abbreviated to $A(\alpha)$. Let $\alpha \subset N$ and $\alpha^{\prime}=N-\alpha$, both arranged in increasing order. Then

$$
A / \alpha=A / A(\alpha)=A\left(\alpha^{\prime}\right)-A\left(\alpha^{\prime}, \alpha\right)[A(\alpha)]^{-1} A\left(\alpha, \alpha^{\prime}\right) .
$$

is called the Schur complement with respect to $A(\alpha)$, and

$$
A / \circ \alpha=A / A(\alpha)=A\left(\alpha^{\prime}\right)-\left\{A\left(\alpha^{\prime}, \alpha\right)[A(\alpha)]^{-1} A\left(\alpha, \alpha^{\prime}\right)\right\} \circ I .
$$

is called the diagonal-Schur complement with respect to $A(\alpha)$, where $A(\alpha)$ is nonsingular and $I$ denote the identity matrix ([5]). Let $A=\left(a_{i j}\right) \in C^{n \times n}$, $n \geq 2$. Denote $R_{i}(A)=\sum_{k \neq i}\left|a_{i k}\right|, \quad i \in N$. Recall that $A$ is called (row) diagonally dominant if

$$
\left|a_{i i}\right| \geq R_{i}(A) \quad i \in N
$$

If all inequalities in (1) are strict, we say that $A$ is a strictly diagonally dominant matrix $\left(A \in S D_{n}\right)$. It is well known that strictly diagonally dominant matrices are nonsingular and the same is true of the principal submatrices of strictly diagonally dominant matrices.

Definition 1 ([3]). A $\in C^{n \times n}$ is called a doubly diagonally dominant matrix (denoted by $A \in D D_{n}$ ) if

$$
\left|a_{i i}\right|\left|a_{j j}\right| \geq R_{i}(A) R_{j}(A), \quad i \neq j ; i, j \in N .
$$


If the inequalities in (2) are strict for all distinct $i, j \in N$, we call $A$ a strictly doubly diagonally dominant matrix $\left(A \in S D D_{n}\right)$.

Obviously, if $A \in S D D_{n}$, there exists at most one index $i_{0}$ such that $\left|a_{i_{0} i_{0}}\right| \leq R_{i_{0}}(A)$. And it is known that $S D_{n} \subset S D D_{n} \subset H_{n}$. Let $A=\left(a_{i j}\right) \in$ $C^{n \times n}, \alpha \subset N$. Define

$$
\begin{gathered}
J_{+}(A)=\left\{i \mid \operatorname{Re} a_{i i}>0\right\}, \quad J_{-}(A)=\left\{i \mid \operatorname{Re} a_{i i}<0\right\}, \\
J_{+}^{\alpha}(A)=\left\{i_{t} \mid \operatorname{Re} a_{i_{t} i_{t}}>0, i_{t} \in \alpha\right\}, \quad J_{-}^{\alpha}(A)=\left\{i_{t} \mid \operatorname{Re} a_{i_{t} i_{t}}<0, i_{t} \in \alpha\right\} .
\end{gathered}
$$

In what follows, we shall show that the diagonal-Schur complements of $H$ matrices are also $H$-matrices, and their distribution of eigenvalues in section 2 . In section 3 we will prove the diagonal-Schur complements of strictly doubly diagonally dominant matrices are also strictly doubly diagonally dominant matrices. Further, we give their distribution of eigenvalues.

\section{Diagonal-Schur complements of $H$-matrices and distribution of eigenvalues}

Let $A=\left(a_{i j}\right) \in R^{n \times n}$ be partitioned as

$$
\left(\begin{array}{ll}
A_{11} & A_{12} \\
A_{21} & A_{22}
\end{array}\right)
$$

where $A_{11}$ is the leading $k \times k$ principal submatrix of $A$, for some $k \in N$.

Lemma 1 ([4]). If $A \in M_{n}, \alpha \subset N$, then $A / \alpha \in M_{n-k}$.

Lemma 2 ([10]). Let $A \in C^{n \times n}, B \in M_{n}, \mu(A) \geq B$. Then $A \in H_{n}$ and $B^{-1} \geq\left|A^{-1}\right| \geq 0$.

Lemma 3 ([4]). If $A \in M_{n}, B \in Z_{n}$ and $B \geq A$, then $B \in M_{n}$.

Lemma 4 ([5]). If $A=\left(a_{i j}\right) \in H_{n}, a_{i i} \in R$ for each $i \in N$ and $a_{i_{i} i_{t}}>$ $0(t=1,2, \ldots, l), a_{i_{u} i_{u}}<0(u=1,2, \ldots, m), l+m=n$, then $A$ has $l$ eigenvalues with positive real part and $m$ eigenvalues with negative real part.

Theorem 1. Let $A \in H_{n}, \alpha \subset N$. Then

(i) $A /{ }_{\circ} \alpha \in H_{n-|\alpha|}$.

(ii) If $a_{i i} \in R$ for each $i \in N, A /{ }_{\circ} \alpha$ has $\left|J_{+}(A)\right|-\left|J_{+}^{\alpha}(A)\right|$ eigenvalues with positive real part and $\left|J_{-}(A)\right|-\left|J_{-}^{\alpha}(A)\right|$ eigenvalues with negative real part.

Proof. Without loss of generality, we can suppose that $A$ has form (3) and 
$\alpha=\{1,2, \ldots, k\}$. Then

$$
\begin{aligned}
& A / \circ A_{11}=A_{22}-\left(A_{21} A_{11}^{-1} A_{12}\right) \circ I=\left(\begin{array}{ccc}
a_{k+1, k+1} & \ldots & a_{k+1, n} \\
\vdots & \ddots & \vdots \\
a_{n, k+1} & \ldots & a_{n n}
\end{array}\right) \\
& -\left(\begin{array}{cccc}
\left(a_{k+1,1} \cdots a_{k+1, k}\right) A_{11}^{-1}\left(\begin{array}{c}
a_{1, k+1} \\
\vdots \\
a_{k, k+1}
\end{array}\right) & & & \\
& & \ddots & \\
& & & \\
0 & & & \left(a_{n, 1} \cdots a_{n, k}\right) A_{11}^{-1}\left(\begin{array}{c}
a_{1, n} \\
\vdots \\
a_{k, n}
\end{array}\right)
\end{array}\right) .
\end{aligned}
$$

(i) Since $A \in H_{n}$, then $\mu(A) \in M_{n}$. By Lemma 1 and Lemma 2, we have

$$
\mu(A) / \mu\left(A_{11}\right) \in M_{n-k}, \quad\left[\mu\left(A_{11}\right)\right]^{-1} \geq\left|A_{11}^{-1}\right| .
$$

For all $j \in\{k+1, \ldots, n\}$,

$$
\begin{aligned}
\left|a_{j j}-\left(a_{j 1} \cdots a_{j k}\right) A_{11}^{-1}\left(\begin{array}{c}
a_{1 j} \\
\vdots \\
a_{k j}
\end{array}\right)\right| & \geq\left|a_{j j}\right|-\left(\left|a_{j 1}\right| \cdots\left|a_{j k}\right|\right)\left|A_{11}^{-1}\right|\left(\begin{array}{c}
\left|a_{1 j}\right| \\
\vdots \\
\left|a_{k j}\right|
\end{array}\right) \\
& \geq\left|a_{j j}\right|-\left(\left|a_{j 1}\right| \cdots\left|a_{j k}\right|\right)\left[\mu\left(A_{11}\right)\right]^{-1}\left(\begin{array}{c}
\left|a_{1 j}\right| \\
\vdots \\
\left|a_{k j}\right|
\end{array}\right) .
\end{aligned}
$$

Thus,

$$
\begin{aligned}
\mu(A) / \mu\left(A_{11}\right) & =\mu\left(A_{22}\right)-\left|A_{21}\right|\left[\mu\left(A_{11}\right)\right]^{-1}\left|A_{12}\right| \leq \mu\left(A_{22}\right)-\left\{\left|A_{21}\right|\left[\mu\left(A_{11}\right)\right]^{-1}\left|A_{12}\right|\right\} \circ I \\
& \leq \mu\left[A_{22}-\left(A_{21} A_{11}^{-1} A_{12}\right) \circ I\right]=\mu\left(A / \circ A_{11}\right) .
\end{aligned}
$$

The first inequality follows since $\left|A_{21}\right|\left[\mu\left(A_{11}\right)\right]^{-1}\left|A_{12}\right| \geq 0$, and the second inequality follows from the inequality (4). By Lemma 3 , we have $\mu\left(A / \circ A_{11}\right) \in$ $M_{n-k}$. Thus, $A /{ }_{\circ} A_{11} \in H_{n-k}$.

(ii) Notice that both $A / A_{11}$ and $A /{ }_{\circ} A_{11}$ have same diagonal entries. By the proof of Theorem 1 in [5], we have

$$
\operatorname{sign} \operatorname{Re}\left\{a_{j j}-\left(a_{j 1} \cdots a_{j k}\right) A_{11}^{-1}\left(\begin{array}{c}
a_{1 j} \\
\vdots \\
a_{k j}
\end{array}\right)\right\}=\operatorname{sign} a_{j j}, \quad j \geq k+1 .
$$

Applying Lemma 4, we obtain (ii).

Corollary 1. If $A \in H_{n}, a_{i i} \in R$ for all $i \in N$. Then $A /{ }_{\circ} J_{-}(A)$ is a positively stable matrix.

Corollary 2. Let $A \in C^{n \times n}, a_{i i} \in R$ for all $i \in N, B \in M_{n}, \alpha \subset N$. If $\mu(A) \geq B$, then $(B / \circ \alpha)^{-1} \geq\left|\left(A /{ }_{\circ} \alpha\right)^{-1}\right| \geq 0$.

Proof. By Theorem 1 and Lemma 4, obviously. 


\section{Diagonal-Schur complements of $S D D_{n}$ and distribution of eigenvalues}

Lemma 5 ([3]). If $A \in S D D_{n}, \alpha \subset N$, then $A / \alpha \in S D D_{n-|\alpha|}$.

Lemma 6 ([3]). If $A \in S D D_{n}$, then $A \in H_{n}$.

Lemma 7 ([5]). Let $A \in H_{n}, \alpha \subset N$. Then

(i) $A / \alpha \in H_{n-|\alpha|}$.

(ii) If $a_{i i} \in R$ for all $i \in N$. Then $A / \alpha$ has $\left|J_{+}(A)\right|-\left|J_{+}^{\alpha}(A)\right|$ eigenvalues with positive real part and $\left|J_{-}(A)\right|-\left|J_{-}^{\alpha}(A)\right|$ eigenvalues with negative real part.

Remark. If $A \in S D D_{n}$, according to Lemma 5, we have $A / \alpha \in S D D_{n-|\alpha|}$. From Lemma 6 , we get that $A / \alpha \in H_{n-|\alpha|}$. Lastly applying Lemma 7 , we obtain $A / \alpha$ have also property (ii) of Lemma 7.

Lemma 8 ([5]). Let $A \in S D_{n}, \alpha \subset N$. Then $A / \circ \alpha \in S D_{n-|\alpha|}$.

Lemma 9 ([11]). (Schur) Let $A \in C^{n \times n}, \alpha \subset N, A(\alpha)$ is nonsingular. Then $\operatorname{det} A=\operatorname{det}(A(\alpha)) \cdot \operatorname{det} A / A(\alpha)$.

Lemma 10. Let $A \in S D D_{n}, J=\left\{i \in N:\left|a_{i i}\right| \leq R_{i}(A)\right\}$ and $A$ be partitioned as form (3). Then

(i) If $\phi \neq J \subset\{1,2, \ldots, k\}, j \in\{k+1, \ldots, n\}$, then

$$
\left|a_{j j}\right|-\left(\left|a_{j 1}\right| \cdots\left|a_{j k}\right|\right)\left[\mu\left(A_{11}\right)\right]^{-1}\left(\begin{array}{c}
\left|a_{1 j}\right| \\
\vdots \\
\left|a_{k j}\right|
\end{array}\right)>\sum_{l=k+1(l \neq j)}^{n}\left|a_{j l}\right| .
$$

(ii) If $\phi \neq J \subset\{k+1, \ldots, n\}$, for all distinct $i, j \in\{k+1, \ldots, n\}$. Then

$$
\begin{aligned}
& {\left[\left|a_{i i}\right|-\left(\left|a_{i 1}\right| \cdots\left|a_{i k}\right|\right)\left[\mu\left(A_{11}\right)\right]^{-1}\left(\begin{array}{c}
\left|a_{1 i}\right| \\
\vdots \\
\left|a_{k i}\right|
\end{array}\right)\right]} \\
& \times\left[\left|a_{j j}\right|-\left(\left|a_{j 1}\right| \cdots\left|a_{j k}\right|\right)\left[\mu\left(A_{11}\right)\right]^{-1}\left(\begin{array}{c}
\left|a_{1 j}\right| \\
\vdots \\
\left|a_{k j}\right|
\end{array}\right)\right]>\sum_{l=k+1(l \neq i)}^{n}\left|a_{i l}\right| \sum_{l=k+1(l \neq j)}^{n}\left|a_{j l}\right| .
\end{aligned}
$$

Proof. (i) Since $A \in S D D_{n}$ and $J \neq \phi$, then there exists only one index $i_{0}$ such that $\left|a_{i_{0} i_{0}}\right| \leq R_{i_{0}}(A)$. Without loss of generality, we assume $i_{0}=1$. By Lemma 9 , for all $j \in\{k+1, \ldots, n\}$,

$$
\left|a_{j j}\right|-\left(\left|a_{j 1}\right| \cdots\left|a_{j k}\right|\right)\left[\mu\left(A_{11}\right)\right]^{-1}\left(\begin{array}{c}
\left|a_{1 j}\right| \\
\vdots \\
\left|a_{k j}\right|
\end{array}\right)-\sum_{l=k+1(l \neq j)}^{n}\left|a_{j l}\right|=\frac{1}{\operatorname{det} \mu\left(A_{11}\right)} \operatorname{det} B
$$




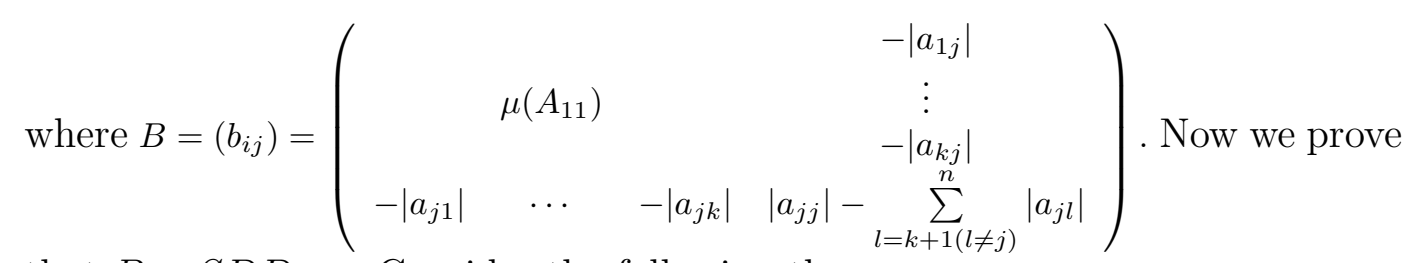
that $B \in S D D_{k+1}$. Consider the following three cases:

Case 1: For all distinct $i, l \in\{1, \ldots, k\}$, since $A \in S D D_{n}$, then the former $k$ rows of $B$ satisfy (2) and the inequality is strict.

Case 2: For $i \in\{2, \ldots, k\} \cup\{j\}$, since $\left|a_{i i}\right|>R_{i}(A)$, then $\left|b_{i i}\right|>R_{i}(B)$.

Case 3: For $i \in\{1\} \cup\{j\}$,

$$
\begin{aligned}
& \left|b_{11} b_{j j}\right|-R_{1}(B) R_{j}(B) \\
& =\left|a_{11}\right|\left(\left|a_{j j}\right|-\sum_{l=k+1(l \neq j)}^{n}\left|a_{j l}\right|\right)-\sum_{l=1}^{k}\left|a_{j l}\right|\left(\sum_{l=2}^{k}\left|a_{1 l}\right|+\left|a_{1 j}\right|\right) \\
& >\sum_{l=1(l \neq j)}^{n}\left|a_{j l}\right| \sum_{l=2}^{n}\left|a_{1 l}\right|-\sum_{l=k+1(l \neq j)}^{n}\left|a_{j l}\right| \sum_{l=2}^{n}\left|a_{1 l}\right|-\sum_{l=1}^{k}\left|a_{j l}\right| \sum_{l=2}^{k}\left|a_{1 l}\right|-\left|a_{1 j}\right| \sum_{l=1}^{k}\left|a_{j l}\right| \\
& =\sum_{l=2}^{n}\left|a_{1 l}\right| \sum_{l=1}^{k}\left|a_{j l}\right|-\sum_{l=1}^{k}\left|a_{j l}\right| \sum_{l=2}^{k}\left|a_{1 l}\right|-\left|a_{1 j}\right| \sum_{l=1}^{k}\left|a_{j l}\right| \\
& =\sum_{l=1}^{k}\left|a_{j l}\right| \sum_{l=k+1}^{n}\left|a_{1 l}\right|-\left|a_{1 j}\right| \sum_{l=1}^{k}\left|a_{j l}\right|=\sum_{l=1}^{k}\left|a_{j l}\right| \sum_{l=k+1(l \neq j)}^{n}\left|a_{1 l}\right| \geq 0 .
\end{aligned}
$$

Above all, we have $B \in S D D_{k+1}$. Applying Lemma 6, $B \in H_{k+1}$. Notice that $B=\mu(B)$, so $B \in M_{k+1}$. Thus $\operatorname{det} B>0$. Obviously, $\mu(A) \in M_{n}$, then $\operatorname{det} \mu\left(A_{11}\right)>0$. Thus we obtain (5).

(ii) $\phi \neq J \subset\{k+1, \ldots, n\}$, without loss of generality, we assume $i_{0}=$ $k+1 \in J$. Consider the following two cases:

Case 1: For all $j \in\{k+2, \ldots, n\}$, by Case 2 in (i), we have

$$
\left|a_{j j}\right|-\left(\left|a_{j 1}\right| \cdots\left|a_{j k}\right|\right)\left[\mu\left(A_{11}\right)\right]^{-1}\left(\begin{array}{c}
\left|a_{1 j}\right| \\
\vdots \\
\left|a_{k j}\right|
\end{array}\right)-\sum_{l=k+1(l \neq j)}^{n}\left|a_{j l}\right|>0 .
$$

Case 2: For all $j \in\{k+2, \ldots, n\}$ and $i_{0}=k+1 \in J$, let

$$
B=\left(\begin{array}{ccccc} 
& & & -\left|a_{1, k+1}\right| & -\left|a_{1 j}\right| \\
& \mu\left(A_{11}\right) & & \vdots & \vdots \\
-\left|a_{k+1,1}\right| & \cdots & -\left|a_{k+1, k}\right| & -\left|a_{k, k+1}\right| & -\left|a_{k j}\right| \\
-\left|a_{j 1}\right| & \cdots & -\left|a_{j k}\right| & -\sum_{l=k+1, k+1}^{n} \mid & -\sum_{l=k+2}^{n}\left|a_{k+1, l}\right| \\
& & & & \left|a_{j, l}\right|
\end{array}\right) .
$$


Since $A \in S D D_{n}$, then $B \in S D D_{k+2}$. Applying Lemma 6, $B \in H_{k+2}$. Notice that $B=\mu(B)$, so $B \in M_{k+2}$. Thus $\operatorname{det} B>0$. Applying Lemma 9,

$$
\operatorname{det} B=\operatorname{det}\left(\mu\left(A_{11}\right)\right) \cdot \operatorname{det} B / \mu\left(A_{11}\right) \text {. }
$$

Since $\mu\left(A_{11}\right) \in M_{k}$, then $\operatorname{det}\left(\mu\left(A_{11}\right)\right)>0$. Therefore,

$$
\begin{aligned}
& \operatorname{det}\left(B / \mu\left(A_{11}\right)\right)>0 . \\
& B / \mu\left(A_{11}\right)=\left(\begin{array}{cc}
\left|a_{k+1, k+1}\right| & -\sum_{l=k+2}^{n}\left|a_{k+1, l}\right| \\
-\sum_{l=k+1(l \neq j)}^{n}\left|a_{j l}\right| & \left|a_{j j}\right|
\end{array}\right) \\
&-\left(\begin{array}{ccc}
\left|a_{k+1,1}\right| & \ldots & \left|a_{k+1, k}\right| \\
\left|a_{j 1}\right| & \ldots & \left|a_{j k}\right|
\end{array}\right)\left[\mu\left(A_{11}\right)\right]^{-1}\left(\begin{array}{cc}
\left|a_{1, k+1}\right| & \left|a_{1 j}\right| \\
\vdots & \vdots \\
\left|a_{k, k+1}\right| & \left|a_{k j}\right|
\end{array}\right) \\
& \triangleq\left(\begin{array}{ccc}
\left|a_{k+1, k+1}\right| & -\sum_{l=k+2}^{n}\left|a_{k+1, l}\right| \\
-\sum_{l=k+1(l \neq j)}^{n} & \left|a_{j l}\right| & \left|a_{j j}\right|
\end{array}\right)-\left(\begin{array}{cc}
c_{11} & * \\
* * & c_{22}
\end{array}\right),
\end{aligned}
$$

where

$c_{11}=\left(\left|a_{k+1,1}\right| \cdots\left|a_{k+1, k}\right|\right)\left[\mu\left(A_{11}\right)\right]^{-1}\left(\begin{array}{c}\left|a_{1, k+1}\right| \\ \vdots \\ \left|a_{k, k+1}\right|\end{array}\right), c_{22}=\left(\left|a_{j, 1}\right| \cdots\left|a_{j k}\right|\right)\left[\mu\left(A_{11}\right)\right]^{-1}\left(\begin{array}{c}\left|a_{1 j}\right| \\ \vdots \\ \left|a_{k j}\right|\end{array}\right)$,

and "*", "**" are nonnegative real numbers.

$$
\begin{aligned}
\operatorname{det} B / \mu\left(A_{11}\right) & =\left[\left|a_{k+1, k+1}\right|-\left(\left|a_{k+1,1}\right| \cdots\left|a_{k+1, k}\right|\right)\left[\mu\left(A_{11}\right)\right]^{-1}\left(\begin{array}{c}
\left|a_{1, k+1}\right| \\
\vdots \\
\left|a_{k, k+1}\right|
\end{array}\right)\right] \\
& \times\left[\left|a_{j j}\right|-\left(\left|a_{j, 1}\right| \cdots\left|a_{j k}\right|\right)\left[\mu\left(A_{11}\right)\right]^{-1}\left(\begin{array}{c}
\left|a_{1 j}\right| \\
\vdots \\
\left|a_{k j}\right|
\end{array}\right)\right] \\
& -\left[\sum_{l=k+2}^{n}\left|a_{k+1, l}\right|+(*)\right]\left[\sum_{l=k+1(l \neq j)}^{n}\left|a_{j l}\right|+(* *)\right]>0 .
\end{aligned}
$$

Notice that both $(*)$ and $(* *)$ are nonnegative real numbers, we obtain $(6)$.

Above all, we completed the proof of (ii).

Applying Lemma 10, we obtain the following Theorem 2. 
Theorem 2. Let $J=\left\{i \in N:\left|a_{i i}\right| \leq R_{i}(A)\right\}$. If $A \in S D D_{n}, \alpha \subset N$. Then

(i) $A /{ }_{\circ} \alpha \in S D_{n-|\alpha|}$ if $J \subset \alpha$.

(ii) $A /{ }_{\circ} \alpha \in S D D_{n-|\alpha|}$ if $\phi \neq J \subset N-\alpha$.

Proof. Without loss of generality, we can assume that $A$ has form (3) and $\alpha=\{1,2, \ldots, k\}$.

(i) If $J=\phi$, by Lemma $8, A / \circ A_{11} \in S D_{n-k}$. For all $\phi \neq J \subset\{1,2, \ldots, k\}$ and $j \in\{k+1, \ldots, n\}$,

$$
\begin{aligned}
& \left|a_{j j}-\left(a_{j 1} \cdots a_{j k}\right) A_{11}^{-1}\left(\begin{array}{c}
a_{1 j} \\
\vdots \\
a_{k j}
\end{array}\right)\right|-\sum_{l=k+1(l \neq j)}^{n}\left|a_{j l}\right| \\
& \geq\left|a_{j j}\right|-\left(\left|a_{j 1}\right| \cdots\left|a_{j k}\right|\right)\left|A_{11}^{-1}\right|\left(\begin{array}{c}
\left|a_{1 j}\right| \\
\vdots \\
\left|a_{k j}\right|
\end{array}\right)-\sum_{l=k+1(l \neq j)}^{n}\left|a_{j l}\right| \\
& \geq\left|a_{j j}\right|-\left(\left|a_{j 1}\right| \cdots\left|a_{j k}\right|\right)\left[\mu\left(A_{11}\right)\right]^{-1}\left(\begin{array}{c}
\left|a_{1 j}\right| \\
\vdots \\
\left|a_{k j}\right|
\end{array}\right)-\sum_{l=k+1(l \neq j)}^{n}\left|a_{j l}\right|>0 .
\end{aligned}
$$

The second inequality follows from $\left[\mu\left(A_{11}\right)\right]^{-1} \geq\left|A_{11}^{-1}\right|$, and the last inequality follows from (i) of Lemma 10. Thus, $A /{ }_{\circ} A_{11} \in S D_{n-k}$. This completes the proof of (i).

(ii) If $\phi \neq J \subset N-\alpha=\{k+1, \ldots, n\}$. For all distinct $i, j \in\{k+1, \ldots, n\}$, we have

$$
\begin{aligned}
& \left|a_{i i}-\left(a_{i 1} \cdots a_{i k}\right) A_{11}^{-1}\left(\begin{array}{c}
a_{1 i} \\
\vdots \\
a_{k i}
\end{array}\right)\right| \cdot\left|a_{j j}-\left(a_{j 1} \cdots a_{j k}\right) A_{11}^{-1}\left(\begin{array}{c}
a_{1 j} \\
\vdots \\
a_{k j}
\end{array}\right)\right|-\sum_{l=k+1(l \neq i)}^{n}\left|a_{i l}\right| \sum_{l=k+1(l \neq j)}^{n}\left|a_{j l}\right| \\
& \geq\left[\left|a_{i i}\right|-\left(\left|a_{i 1}\right| \cdots\left|a_{i k}\right|\right)\left|A_{11}^{-1}\right|\left(\begin{array}{c}
\mid \\
\vdots \\
\left|a_{k i}\right|
\end{array}\right)\right] \times\left[\left|a_{j j}\right|-\left(\left|a_{j 1}\right| \cdots\left|a_{j k}\right|\right)\left|A_{11}^{-1}\right|\left(\begin{array}{c}
\left|a_{1 j}\right| \\
\vdots \\
\left|a_{k j}\right|
\end{array}\right)\right] \\
& \geq \quad-\sum_{l=k+1(l \neq i)}^{n}\left|a_{i l}\right| \sum_{l=k+1(l \neq j)}^{n}\left|a_{j l}\right| \\
& \times\left[\left|a_{i i}\right|-\left(\left|a_{i 1}\right| \cdots\left|a_{i k}\right|\right)\left[\mu\left(A_{11}\right)\right]^{-1}\left(\begin{array}{c}
\left|a_{1 i}\right| \\
\vdots \\
\left|a_{k i}\right|
\end{array}\right)\right] \\
& \times\left[\left|a_{j j}\right|-\left(\left|a_{j 1}\right| \cdots\left|a_{j k}\right|\right)\left[\mu\left(A_{11}\right)\right]^{-1}\left(\begin{array}{c}
\left|a_{1 j}\right| \\
\vdots \\
\left|a_{k j}\right|
\end{array}\right)\right]-\sum_{l=k+1(l \neq i)}^{n}\left|a_{i l}\right| \sum_{l=k+1(l \neq j)}^{n}\left|a_{j l}\right|>0 .
\end{aligned}
$$

The second inequality follows from $\left[\mu\left(A_{11}\right)\right]^{-1} \geq\left|A_{11}^{-1}\right|$, and the last inequality follows from (ii) of Lemma 10. Thus, $A / \circ A_{11} \in S D D_{n-k}$. This completes the proof of (ii). 
Corollary 3. Let $A \in S D D_{n},\left|a_{i i}\right| \leq R_{i}(A)$. Then

(i) $A / \circ\left\{a_{i i}\right\} \in S D_{n-1}$.

(ii) For all $j \in N-\{i\}, A / \circ\left\{a_{j j}\right\} \in S D D_{n-1}$.

Corollary 4. Let $A \in S D D_{n}$. Then $|\operatorname{det} A| \geq \prod_{i=1}^{n}|| a_{i i}\left|-R_{i}(A)\right|$.

Proof. Since $A \in S D D_{n}$, then $\mu(A) \in M_{n}$. For arbitrary constant $0<\varepsilon<$ $\min _{1 \leq i \leq n}\left\{|| a_{i i}\left|-R_{i}(A)\right|\right\}$, set $D=\operatorname{diag}\left(|| a_{11}\left|-R_{1}(A)\right|, \cdots,|| a_{n n}\left|-R_{n}(A)\right|\right)$ and $\widetilde{A}=\left(\begin{array}{cc}\mu(A) & -(D-\varepsilon I) \\ -(D-\varepsilon I) & D\end{array}\right)$. By calculation, we have $\widetilde{A} \in S D D_{2 n} \cap M_{2 n}$. And applying Theorem 2, $\widetilde{A} /{ }_{\circ} D=\mu(A)-D^{-1}(D-\varepsilon I)^{2} \in S D D_{n} \cap M_{n}$. In a manner similar to the proof of Corollary 5 in [5], one can obtain our results.

Theorem 3. Let $A \in S D D_{n}, \alpha \subset N$. If $a_{i i} \in R$ for each $i \in N$, Then $A / \circ \alpha$ has $\left|J_{+}(A)\right|-\left|J_{+}^{\alpha}(A)\right|$ eigenvalues with positive real part and $\left|J_{-}(A)\right|-$ $\left|J_{-}^{\alpha}(A)\right|$ eigenvalues with negative real part.

Proof. Applying Theorem 2, $A /{ }_{\circ} \alpha \in S D D_{n-|\alpha|}$. Thus $A /{ }_{\circ} \alpha \in H_{n-|\alpha|}$. We obtain our results from Theorem 1.

Corollary 5. If $A \in S D D_{n}$, with $a_{i i} \in R$ for each $i \in N$. Then $A /{ }_{\circ} J_{-}(A)$ is a positively stable matrix.

Proof. We obtain our results from Theorem 3.

Now we turn our attention to diagonal-Schur complements of $D D_{n}$.

Theorem 4. If $A \in D D_{n}, \alpha \subset N$ with $\operatorname{det} A(\alpha) \neq 0$. Then $A / \circ \alpha \in$ $D D_{n-|\alpha|}$.

Proof. Without loss of generality, we can assume that $A$ has form (3) and $\alpha=\{1,2, \ldots, k\}$. Since $\operatorname{det} A_{11} \neq 0$, by the proof of Theorem 3.7 in [3], we have $a_{i i} \neq 0$ for all $i \in\{1,2, \ldots, k\}$. Let $D=\operatorname{diag}\left(\delta_{1}, \delta_{2}, \cdots, \delta_{n}\right)$, where, for all $i \in\{1,2, \ldots, n\}, \delta_{i}=\left\{\begin{array}{ll}1, & \operatorname{Re} a_{i i} \geq 0, \\ -1, & \operatorname{Re} a_{i i}<0 .\end{array}\right.$ Note that $A+\varepsilon D \in S D D_{n}$ for arbitrary constant $\varepsilon>0$, and $A(\varepsilon)=A+\varepsilon D=\left(\begin{array}{cc}A_{11}(\varepsilon) & A_{12} \\ A_{21} & A_{22}(\varepsilon)\end{array}\right)$. By Theorem 2, we get $A(\varepsilon) /{ }_{\circ} A_{11}(\varepsilon) \in S D D_{n-k}$. Thus, $A(\varepsilon) / \circ A_{11}(\varepsilon)$ satisfies either the inequality (7) or the inequality (8), that is, for all distinct $i, j \in$ $\{k+1, \ldots, n\}$, either

$$
\left|a_{j j}+\varepsilon \delta_{j}-\left(a_{j 1} \cdots a_{j k}\right)\left[A_{11}(\varepsilon)\right]^{-1}\left(\begin{array}{c}
a_{1 j} \\
\vdots \\
a_{k j}
\end{array}\right)\right|-\sum_{l=k+1(l \neq j)}^{n}\left|a_{j l}\right|>0,
$$


or

$$
\begin{aligned}
& \left|a_{i i}+\varepsilon \delta_{i}-\left(a_{i 1} \cdots a_{i k}\right)\left[A_{11}(\varepsilon)\right]^{-1}\left(\begin{array}{c}
a_{1 i} \\
\vdots \\
a_{k i}
\end{array}\right)\right| \\
& \times\left|a_{j j}+\varepsilon \delta_{j}-\left(a_{j 1} \cdots a_{j k}\right)\left[A_{11}(\varepsilon)\right]^{-1}\left(\begin{array}{c}
a_{1 j} \\
\vdots \\
a_{k j}
\end{array}\right)\right|-\sum_{l=k+1(l \neq i)}^{n}\left|a_{i l}\right| \sum_{l=k+1(l \neq j)}^{n}\left|a_{j l}\right|>0 .
\end{aligned}
$$

Since $\lim _{\varepsilon \rightarrow 0}\left[A_{11}(\varepsilon)\right]^{-1}=A_{11}^{-1}$, and by taking the limitation in the inequality (9) and (10) as $\varepsilon \rightarrow 0$, we obtain our results.

\section{References}

[1] D. E. Crabtree, Application of $M$-matrices to nonnegative matrices, Duke Math. J., 33 (1966) 197-208.

[2] R. L. Smith, Some interlacing properties of the Schur complements of a Hermition matrix, Lin. Alg. Appl., 177 (1992) 137-144.

[3] B. Li, M. Tsatsomeros, Doubly diagonally dominant matrices, Lin. Alg. Appl., 261 (1997) 221-235.

[4] D. Carlson, T. Markham, Schur complements of diagonally dominant matrices, Czech. Math. J., 29(104)(1979) 246-251.

[5] J. Liu, Y. Huang, Some properties on Schur complements of $H$-matrices and diagonally dominant matricees, Lin. Alg. Appl., 389 (2004) 365-380.

[6] C. R. Johnson, Inverse M-mareices, Lin. Alg. Appl., 47(1982) 195-216.

[7] J. Liu, Y. Huang, F-Z. Zhang, The Schur complements of generalized doubly diagonally dominant matrices, Lin. Alg. Appl., 378 (2004) 231-244.

[8] R. A. Horn, C. R. Johnson, Matrix Analysis, Cambridge University Press, New York, 1985.

[9] R. A. Horn, C. R. Johnson, Topics in Matrix Analysis, Cambridge University Press, New York, 1991.

[10] D. E. Crabtree, E. V. Haynsworth, An identity for the Schur complement of a matrix, Proc. Amer. Math. Soc., 22 (1969) 364-366.

[11] F.-Z. Zhang, Matrix Theory: Basic Results and Techniques, Springer, New York, 1999.

\section{Received: May 14, 2006}

